\title{
Optimal Integration of Intermittent Energy Sources Using Distributed Multi-Step Optimization
}

\author{
Kyri Baker, Student Member, IEEE, Gabriela Hug, Member, IEEE, and Xin Li, Senior Member, IEEE.
}

\begin{abstract}
The integration of renewable energy sources such as wind and solar into the electric power grid is a coveted yet challenging goal. The difficulties arise from the intermittency of the sources, the required increase in transmission capacity, and the lack of coordination between control entities. In this paper, a method is developed and implemented for the optimal coordination between storage and intermittent resources in multiple control areas. The problem is formulated as a decomposed multi-step optimization problem using the Optimality Condition Decomposition method. This allows reducing the computational effort by dividing the overall optimization problem into subproblems. Simulation results show convergence to the centralized solution and provide an indication of the benefits of coordinating control areas.
\end{abstract}

\section{INTRODUCTION}

$\mathbf{T}$ RADITIONAL energy generation from sources such as fossil fuel power plants is harmful to the environment as well as non-sustainable. It is desirable to replace these generation plants by renewable sources such as wind power, but these sources are intermittent and non-dispatchable. Another challenge is that in the United States, the locations with the highest wind speeds are located in the Midwest but load centers are mainly on the coasts, and the existing grid infrastructure was not designed for transmitting significant power from these locations to the coasts. This situation is complicated by the fact that there is a lack of coordination between control areas, resulting in a non-optimal usage of available balancing resources. In order to reach the US Department of Energy's goal of $20 \%$ wind penetration by 2030 [1], a solution to these problems must be obtained.

Some goals of the "smart grid", a redesigned power grid, are to provide better control over the power flow in the grid, monitor the system state more accurately, and increase communication capabilities within the grid. These features will allow better usage of the existing system infrastructure and efficiently integrate new elements such as renewable generation and/or storage devices into the system.

This paper focuses on how to optimally coordinate available storage capabilities in one control area with intermittent renewable generation in another area to reliably supply the requested load. By using storage located near load centers, it is possible to transmit the produced power to these locations during times when the transmission system is not heavily loaded and feed it into the storage devices. This energy can then be used during high load situations when the transmission capacity is

The authors are with the Department of Electrical and Computer Engineering, Carnegie Mellon University, Pittsburgh, PA, 15213 USA. E-mail: kyri@cmu.edu,ghug@ece.cmu.edu,xinli@ece.cmu.edu. at its limit and the demand exceeds the supply, alleviating the problem of underrated infrastructure. The intermittency issue is addressed as well, as storage also allows balancing the intermittent power output from the renewable generators.

With storage devices in the system, the question arises of when to store and when to withdraw energy from the device. In this paper, we will use a multi-step optimization based on model predictive control for optimal usage of the storage device because it allows taking into account wind forecasts and explicitly incorporates capacity constraints of the storage device.

Thus, optimization does not just take place over a single time step but over an entire time horizon. The consequence is that the number of variables and problem size grows greatly. It becomes challenging to apply this computationally intensive technique to a large system with fast time constants such as a power system. This, along with the fact that the control decisions in the system are made across separate control areas, makes using a mathematical decomposition algorithm very valuable. Optimality Condition Decomposition [4], a technique based on Lagrangian Relaxation, in combination with the Unlimited Point Algorithm, is used to decompose and efficiently solve the overall optimization problem.

The paper is organized as follows: Section II discusses related work in this area. Section III discusses how the generators, load, storage, and system constraints are modeled. In Section IV, the background for the methodologies used in the algorithm are explained. Simulation results and discussion are given in Section $\mathrm{V}$ and concluding remarks and plans for future work are discussed in Section VI.

\section{Previous Related Work}

The multi-step receding horizon optimization used in this paper is based on model predictive control (MPC). MPC has been previously applied to the field of power systems. In [8], intermittent resources are coordinated with conventional generators to minimize an economic dispatch function subject to DC power flow equations. Centralized control is implemented, and there are no nonlinear constraints on the system.

A distributed model predictive control algorithm is developed in [9], and is applied to linear, time-invariant systems. An interior-point/barrier method is used for the optimization, and each agent uses the same set of Lagrange multipliers. A two-area power system load-frequency control application is used to illustrate that the algorithm achieves the same solution as the centralized problem.

Distributed MPC is applied to power systems in [10] for the mitigation of cascading failures, and uses models of 
neighboring areas. The control agents may anticipate that neighboring areas will make different control decisions than those calculated by the other agents themselves according to the accuracy of these models. The agents exchange these decisions with neighbors to minimize the differences.

In [11], distributed MPC is applied to automatic generation control. A communication-based MPC technique is discussed where each area optimizes its own cost function until a Nash Equilibrium is achieved. Along with this approach, a cooperation-based MPC approach is discussed that achieves the same solution as the centralized case for achieving systemwide objectives. Local controller cost functions are replaced by objective functions that measure the systemwide impact of local actions.

Two methods used in conjunction in this paper, Optimality Condition Decomposition and Unlimited Point Algorithm, were both used in [12] to achieve coordinated power flow control using FACTS (Flexible AC Transmission Systems) devices for the enhancement of power system steady-state security.

The method we develop in this paper optimizes an economic and environmental dispatch objective utilizing distributed multi-step optimization. The advantages of the employed decomposition technique is that no parameter tuning, other than step size damping, is necessary; fast convergence is achieved, and it is computationally inexpensive. Furthermore, no approximate models of neighboring areas are required and AC power flow is incorporated.

Power systems, in general, are large-scale systems with hundreds or thousands of variables. With the developed technique, the computational effort is greatly reduced. Only one Newton-Raphson iteration is performed for each subproblem before the areas are required to exchange data. In other distributed MPC techniques, the subproblems must be solved to optimality. A central controller is not needed, unlike in Lagrangian Relaxation, for example, where a central controller is responsible for updating the Lagrange multipliers.

\section{System Modeling}

\section{A. Problem Formulation for Economic Dispatch and Emission Minimization}

The objective in this paper is to increase the penetration of renewable resources, with the ultimate goal of reducing harmful emissions and the cost of traditional generation. This objective is modeled using the following multi-objective economic and environmental dispatch cost function:

$$
f=\sum_{i=1}^{n u m G e n} a_{i} P_{G_{i}}^{2}+b_{i} P_{G_{i}}+c_{i}+\eta_{i} P_{G_{i}}^{2}+\xi_{i} P_{G i},
$$

where $a_{i}, b_{i}, c_{i}$ are the penalizing coefficients for active power generation from generator $i$, and $\eta_{i}$ and $\xi_{i}$ are penalizing coefficients related to generator $i$ 's emissions cost. The optimization control variables include the active power generation, and power flow into/out of storage.

\section{B. Storage}

The utilization of storage is a key component to the integration of intermittent resources. Firstly, it provides a balance to the intermittency of renewables. Directly using the power output of a wind generator will result in highly fluctuating generation levels, which means the conventional generators must consequently ramp up and down to provide an overall constant level of power. Secondly, if the transmission lines are heavily loaded and additional power is needed to be transmitted to a load, having the option of storage nearby is highly advantageous. Power can be transmitted to the storage during times when the lines are lightly loaded, and that stored power can be used to supply the load as needed during times when the power lines are near capacity. The model of storage used in this paper is depicted in Figure 1.

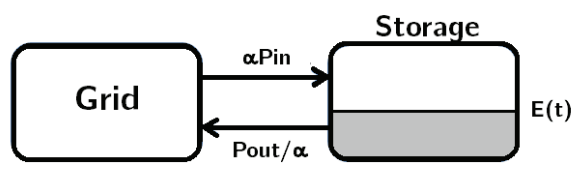

Fig. 1. Model for storage

Storage devices in the system are modeled as having a current amount of stored energy $E$ and a roundtrip efficiency of $\alpha^{2}$. Defined also is the time scale, $T$, the time between control decisions. The constraints on storage are as follows:

$$
\begin{aligned}
E(t+T) & =E(t)+\alpha T P_{\text {in }}(t)-\frac{T}{\alpha} P_{\text {out }}(t), \\
E^{\text {min }} & \leq E(t+T) \leq E^{\text {max }} \\
0 & \leq P_{\text {in }}(t) \leq P_{\text {in }}^{\max } \\
0 & \leq P_{\text {out }}(t) \leq P_{\text {out }}^{\max }
\end{aligned}
$$

Note that a constraint preventing the simultaneous charging/discharging of the storage device, $P_{\text {out }}(t) P_{\text {in }}(t)=0$ is omitted here. This constraint is unnecessary for the given objective function and problem setup; only in the unrealistic case where the efficiency $\alpha=1$, the optimizer will never find it optimal to charge and discharge at the same time, because energy will only be lost.

\section{System Equations}

Along with the constraints on storage, constraints on power balance and generators are considered. The following variables are defined:

$\begin{array}{rr}P_{G k}: & \text { Active power generation at bus } k \\ Q_{G k}: & \text { Reactive power generation at bus } k \\ P_{W k}: & \text { Available wind at bus } k \\ P_{L k}: & \text { Active power consumption at bus } k \\ Q_{L k}: & \text { Reactive power consumption at bus } k \\ P_{k}: & \text { Active power injected into system at bus } k \\ Q_{k}: & \text { Reactive power injected into system at bus } k\end{array}$




$$
\begin{array}{rr}
P_{\text {in }, k}: & \text { Power put into storage at bus } k \\
P_{\text {out }, k}: & \text { Power withdrawn from storage at bus } k
\end{array}
$$

The constraints on power balance and generators used in this paper is as follows:

$$
\begin{aligned}
P_{k}-P_{G k}+P_{L k}-P_{W k}-P_{i n, k}+P_{o u t, k} & =0, \\
Q_{k}-Q_{G k}+Q_{L k} & =0, \\
P_{G i} & \geq 0,
\end{aligned}
$$

with the well-known power balance equations at bus $k$ defined as:

$$
\begin{aligned}
& P_{k}=\left|V_{k}\right| \sum_{m \in \Omega_{k}}\left|V_{m}\right|\left(G_{k m} \cos \theta_{k m}+B_{k m} \sin \theta_{k m}\right), \\
& Q_{k}=\left|V_{k}\right| \sum_{m \in \Omega_{k}}\left|V_{m}\right|\left(G_{k m} \sin \theta_{k m}-B_{k m} \cos \theta_{k m}\right),
\end{aligned}
$$

where $\Omega_{k}$ is the set of all buses connected to bus $k$ (including bus $k$ itself), $\left|V_{k}\right|$ is the voltage magnitude at bus $k, \theta_{k m}$ is the voltage angle difference between buses $k$ and $m, G_{k m}$ is the real part of the admittance matrix element $y_{k m}$, and $B_{k m}$ is the imaginary part of $y_{k m}$.

\section{Methodologies}

\section{A. Model Predictive Control}

The look-ahead optimization procedure used in this paper is derived from Model Predictive Control (MPC). MPC, also called receding horizon control, shown in Figure 2, minimizes the cost of control decisions on a system over a prediction horizon $N$. This is done by forming a model of the system to be controlled and optimizing over a chosen number of time steps in the future using the predicted output of the system. After this optimization from discrete times $t$ to $t+N$ is complete, only the actions for time $t$ are applied. Measurements from the actual system are then taken, the model is updated, and the optimization is recalculated for the next time step [2].

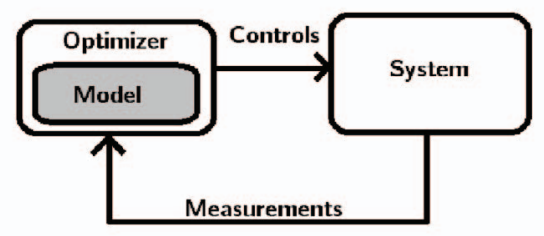

Fig. 2. Visual representation of Model Predictive Control.

The model of the system used is described in the previous section, with models for the wind and load curve predictions. The formulation for a discrete-time, nonlinear MPC problem is as follows [3]:

$$
\begin{array}{cl}
\underset{x, u}{\operatorname{minimize}} & \sum_{t=1}^{N} J(x(t), u(t)) \\
\text { subject to } & g(x(t), u(t))=0, t=1 \ldots N \\
& h(x(t), u(t)) \leq 0, t=1 \ldots N \\
& x(t+1)=f(x(t), u(t)), t=0 \ldots N-1,
\end{array}
$$

with state variables $x$ and input variables $u$. Optimal values are calculated for the entire horizon but only the first step is applied. The simulation moves to the next step and the process is repeated using updated measurements of the system state. The multi-step optimization procedure is based on this methodology, but differs from traditional MPC; power systems are modeled using steady-state equations rather than the dynamic equations typically used to model control systems in MPC. In addition, traditional MPC seeks to minimize prediction errors; we currently do not correct wind or demand prediction errors. Developing an improved prediction scheme with reduced errors will be considered as our future work.

\section{B. Optimality Condition Decomposition}

Decomposing an optimization problem has multiple advantages. In large-scale systems such as power systems, multiple entities share the responsibility of controlling the system. A centralized controller may not exist or entities may not be willing to exchange extensive data. Decomposing problem also allows for ease of parallel computations for the subproblems; to perform calculations on one large centralized system results in a higher computational complexity than parallelizing the calculations over multiple smaller subproblems.

In this paper, Optimality Condition Decomposition [4], a modified version of Lagrangian Relaxation Decomposition, is used. Using this decomposition method, separability is achieved by fixing certain coupled variables as constants during each iteration step. The general optimization problem can be formulated as follows:

$$
\begin{aligned}
& \underset{x_{1}, \ldots, x_{P}}{\operatorname{minimize}} f\left(x_{1}, \ldots, x_{P}\right) \\
& \text { subject to } g_{p}\left(x_{p}\right)=0, p \in\{1, \ldots, P\} \\
& h_{p}\left(x_{p}\right) \leq 0, p \in\{1, \ldots, P\} \\
& g_{p, \text { coup }}\left(x_{1}, \ldots, x_{P}\right)=0, p \in\{1, \ldots, P\} \\
& h_{p, \text { coup }}\left(x_{1}, \ldots, x_{P}\right) \leq 0, p \in\{1, \ldots, P\}
\end{aligned}
$$

where $P$ is the total number of subproblems and $x_{p}$ are the decision variables in subproblem $p$, where $p \in\{1, \ldots, P\}$. $g_{p}$ and $h_{p}$ are constraints dependent only on variables $x_{p}$ in subproblem $p . g_{p, \text { coup }}$ and $h_{p, \text { coup }}$ are called "coupling constraints" corresponding to constraints that contain variables from multiple subproblems.

Optimality Condition Decomposition decomposes this optimization problem by assigning variables and constraints to specific subproblems. Every coupling constraint is assigned to one specific subproblem which takes this constraint into account as a hard constraint in its constraint set. This coupling constraint is taken into account as a soft constraint in the objective function of the other subproblems. If a subproblem contains a constraint with a variable belonging to another subproblem, that so-called "foreign" variable is set to a constant value given by its corresponding subproblem. This value is updated at the next iteration when the subproblems exchange these coupled variables. An optimization problem is formed for each subproblem $p \in\{1 \ldots P\}$ as follows: 


$$
\begin{gathered}
\underset{x_{p}}{\operatorname{minimize}} f_{p}=\left(f\left(\bar{x}_{1}, \ldots, \bar{x}_{p-1}, x_{p}, \bar{x}_{p+1}, \ldots, \bar{x}_{P}\right)\right. \\
+\sum_{n=1, n \neq p}^{P} \bar{\lambda}_{n} g_{n, \text { coup }}\left(\bar{x}_{1}, \ldots, \bar{x}_{p-1}, x_{p}, \bar{x}_{p+1}, \ldots, \bar{x}_{P}\right) \\
\left.+\sum_{n=1, n \neq p}^{P} \bar{\mu}_{n} h_{n, \text { coup }}\left(\bar{x}_{1}, \ldots, \bar{x}_{p-1}, x_{p}, \bar{x}_{p+1}, \ldots, \bar{x}_{P}\right)\right)
\end{gathered}
$$

subject to

$$
\begin{aligned}
g_{p}\left(x_{p}\right) & =0, \\
h_{p}\left(x_{p}\right) & \leq 0, \\
g_{p, \text { coup }}\left(\bar{x}_{1}, \ldots, x_{p}, \ldots, \bar{x}_{P}\right) & =0, \\
\left.h_{p, \text { coup }}\left(\bar{x}_{1}, \ldots, x_{p}, \ldots, \bar{x}_{P}\right)\right) & \leq 0 .
\end{aligned}
$$

Variables denoted with an overhead bar are foreign variables, set to constant values given by their corresponding subproblem, and variables without the bar are the optimization variables of that subproblem. $\lambda_{n}$ and $\mu_{n}$ are the Lagrange multipliers for the equality and inequality constraints (respectively) determined by the subproblem $n$ for which this constraint is a hard constraint. An outline of this iterative procedure is as follows:

1) Assign constraints and variables to a specific subproblem.

2) Initialize variables $\bar{x}_{p}$ and Lagrange multipliers $\bar{\lambda}_{p}, \bar{\mu}_{p}$ for $p \in\{1, \ldots, P\}$.

3) Formulate the first order optimality conditions for each subproblem and perform one Newton-Raphson step.

4) Update $\bar{x}_{p}, \bar{\lambda}_{p}$, and $\bar{\mu}_{p}$ with the values obtained in the previous step.

5) If stopping criteria is fulfilled, stop. Otherwise, go to step 2.

A major advantage of this decomposition over others using Lagrangian techniques is that no parameter tuning is needed and updates for the Lagrange multipliers do not have to be estimated - they are explicitly given from the solutions of the subproblems that they belong to. In power systems, assigning variables and constraints to subproblems is very straightforward; subproblems are defined as physical areas which contain certain buses, so the variables and constraints associated with each bus are automatically assigned to the subproblem/area that bus is in. Another, perhaps greater improvement is that the subproblems do not need to be solved until optimality; one Newton-Raphson iteration is performed, the subproblems exchange information, and then the process is repeated. This results in a significant improvement in computational efficiency, and differs from other Lagrange decompositions that require the subproblems to be solved entirely in order to obtain updates for the Lagrange multipliers. The optimality conditions that must be fulfilled for convergence are the same as that of the centralized problem, and the distributed algorithm will converge to the same solution as in the centralized case given that the requirements on spectral radius described in [4] hold.

\section{Unlimited Point Algorithm}

We will employ the Unlimited Point Algorithm to solve the constrained optimization problem. This method provides a way to formulate the first order optimality conditions such that they do not contain any inequalities. The Lagrangian function is formed for subproblem $p$ :

$$
\mathcal{L}_{p}(x)=f_{p}(x)+\lambda_{p}^{T} g_{p}(x)+\mu_{p}^{T} h_{p}(x),
$$

where $\lambda_{p}$ and $\mu_{p}$ are the Lagrange multipliers for the equality and inequality constraints, respectively, for subproblem $p$. The Karush-Kuhn-Tucker (KKT) conditions [7], i.e., the firstorder conditions for optimality, are formed and the inequality constraints are transformed to equalities using slack variables $z$ :

$$
h_{p}(x)+z_{p}(x)=0,
$$

with non-negativity constraints $\mu_{p} \geq 0, z_{p} \geq 0$ for $\mu$ and $z$. The Unlimited Point Algorithm [5] offers a simple method to ensure these constraints are fulfilled without having to implement penalty or barrier terms. By raising $\mu$ and $z$ to an even power, it ensures that they are always positive - the feasible region is now "unlimited."

After this transformation, the final optimality conditions become:

$$
\begin{aligned}
\frac{\partial}{\partial x}\left(f_{p}(x)+\hat{\lambda}_{p}^{T} g_{p}(x)+\left.\hat{\mu}_{p}^{2 s^{T}}\left(h_{p}(x)+z_{p}^{2 r}\right)\right|_{\hat{x}}\right. & =0, \\
g_{p}(\hat{x})= & 0, \\
h_{p}(\hat{x})+\hat{z}_{p}^{2 r}= & 0, \\
\hat{\mu}_{p} \hat{z}_{p}=0, & 0,
\end{aligned}
$$

where integers $s, r>0$.

Faster convergence may be achieved by increasing $s$ and $r$, however, making these parameters too large may result in divergence. The simulations in this paper do not deal with tuning these parameters for faster convergence and only use $s=r=1$.

The form of the Jacobian matrix, $J_{p}$, of the partial derivatives of the KKT conditions with respect to the optimization variables, Lagrange multipliers, and slack variables in subproblem $p$ is shown below (the subscript $p$ is omitted here for readability):

$$
\left[\begin{array}{cccc}
\nabla^{2} \mathcal{L}(x) & \nabla g(x) & \nabla h(x)^{T} \operatorname{diag}\left\{2 s \mu^{2 s-1}\right\} & 0 \\
\nabla g(x) & 0 & 0 & 0 \\
\nabla h(x) & 0 & 0 & \operatorname{diag}\left\{2 r z^{2 r-1}\right\} \\
0 & 0 & \operatorname{diag}\{z\} & \operatorname{diag}\{\mu\}
\end{array}\right]
$$

After each iteration, data is exchanged between subproblems and the foreign variables are updated to new constant values. The optimization stops when the convergence criteria is fulfilled for every subproblem. The $K K T_{p}$ vector is defined as a vector containing the first order conditions for optimality for subproblem $p$. The convergence criteria for subproblem $p$ is that the Euclidean norm of $K K T_{p}$ must be less than some tolerance $\epsilon$ : 


$$
\left\|K K T_{p}\right\|_{2}<\epsilon .
$$

Overall system convergence is achieved when this condition is fulfilled for all subproblems. Cases where a solution does not exist can occur; if, for example, the power flow equations cannot be fulfilled - but we have yet to encounter such a situation for our problem setup and therefore such considerations are beyond the scope of this paper.

Solving the decomposed optimization problem over several time steps greatly increases the computational complexity; the number of variables per optimization is multiplied by the horizon length $N$. The advantage of having to perform only one Newton-Raphson iteration before exchanging data between subproblems is now even more evident. A flowchart of the final algorithm is shown in Figure 3.

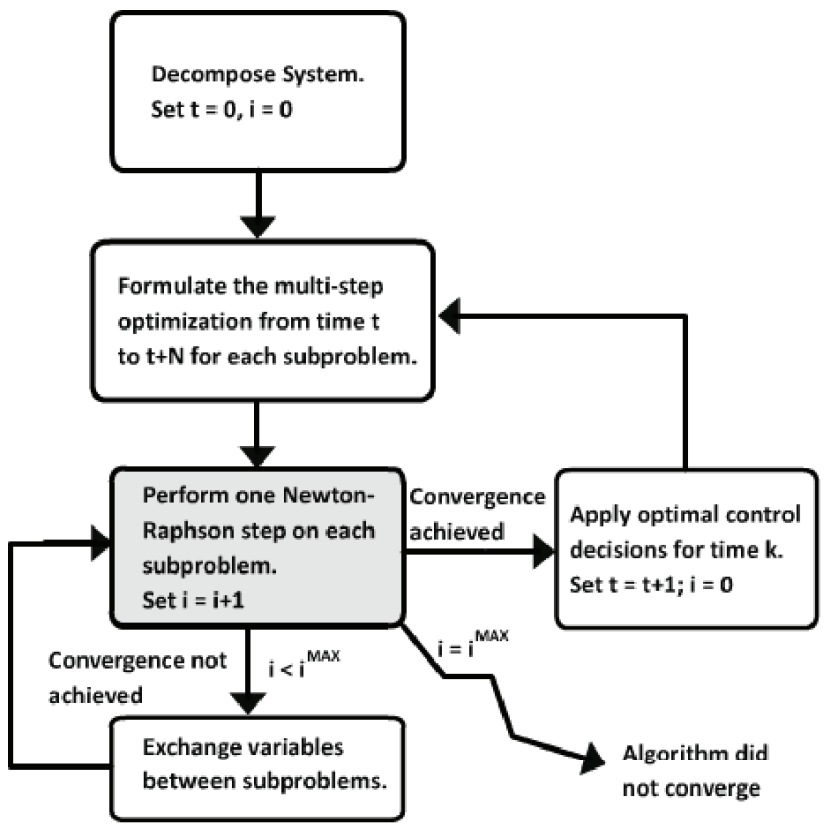

Fig. 3. Flowchart of the distributed optimization algorithm

\section{Simulation Results and Discussion}

\section{A. Data Sets and Results}

Simulations were run on the IEEE 14-bus system [6]. This system contains two generators and three synchronous condensers (which exclusively generate reactive power). The system has been modified to include wind generators at buses 5 and 14 and a storage device at bus 5 . The synchronous condenser at bus 3 has been changed to additionally generate active power, and all of the line parameters were kept as stated in the IEEE specifications. The modified system is shown in Figure 4.

Table I illustrates the values in the objective function (1) chosen for generators in the simulations. The storage device was modeled to have roundtrip efficiency $\alpha^{2}=0.95$, a minimum state-of-charge of $0.2 p . u . \cdot 5$-minutes, and a maximum state-of-charge as $1.5 p . u . \cdot 5$-minutes; i.e., a fairly small amount of storage compared to the total system load.

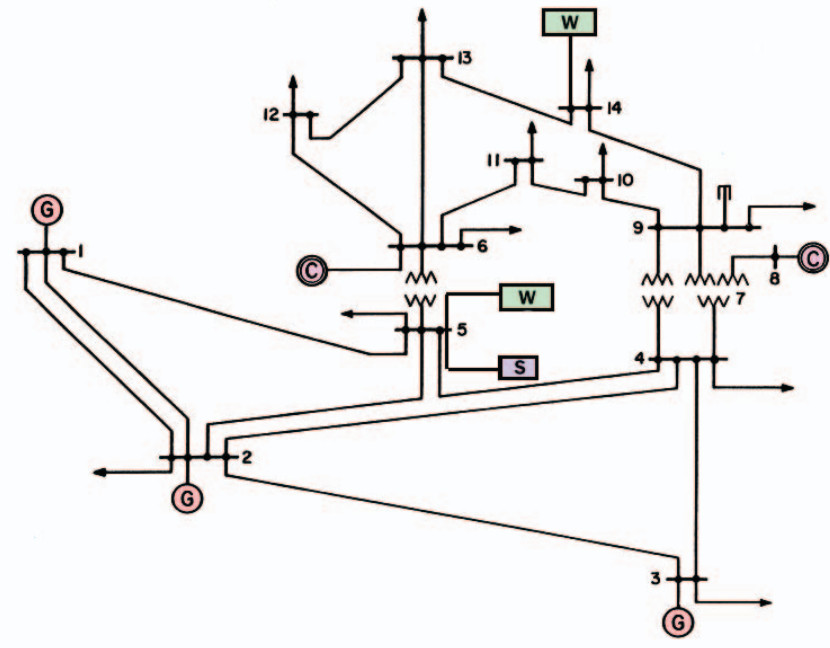

Fig. 4. Modified IEEE 14-bus test system.

TABLE I

Generator PARAMETERS

\begin{tabular}{|l|c|c|c|c|c|}
\hline Generator & $a_{i}$ & $b_{i}$ & $c_{i}$ & $\eta_{i}$ & $\xi_{i}$ \\
\hline$P_{G 1}$ & 0.4 & 1 & 0 & 0.3 & 1 \\
\hline$P_{G 2}$ & 0.5 & 1 & 0 & 0.3 & 1 \\
\hline$P_{G 3}$ & 0.6 & 1.5 & 0 & 0.3 & 1 \\
\hline
\end{tabular}

The wind and load data for these simulations was obtained from the Bonneville Power Administration [14] forecasts for 2011. The data samples are given in 5-minute increments and the simulations were run over a period of 200 samples (1000 minutes; 16.67 hours). In the simulations in this paper, wind and load forecasts are assumed to be equal to their predicted values for times up to $N=10$ (50 minutes). The value for the base power used to convert the units to per-unit is $S_{b}=$ $100 M W$. The data used for the wind and load are shown in Figure 5.

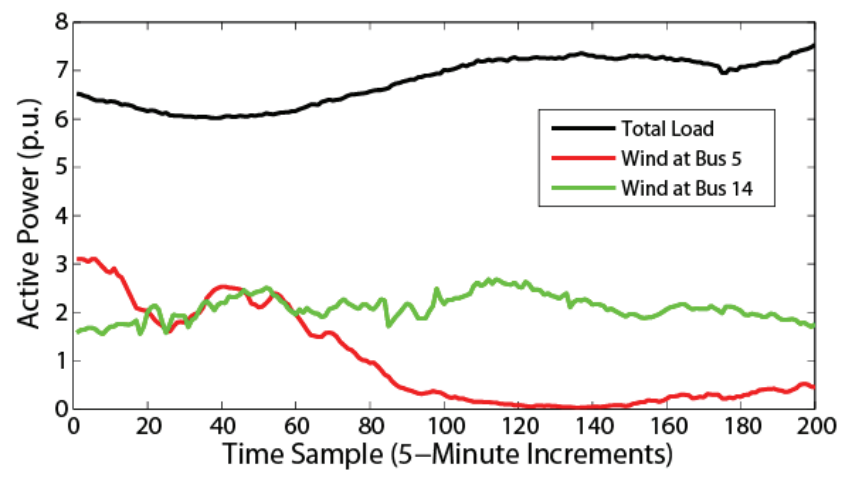

Fig. 5. Total system demand and available wind

In Figure 6, the generation values with no storage in the system are shown.

The system was decomposed into two subproblems with the following sets of buses for each subproblem as seen in (13) and Figure 7. The coupling variables, i.e., the voltage magnitudes and angles at the border buses as well as the Lagrange 


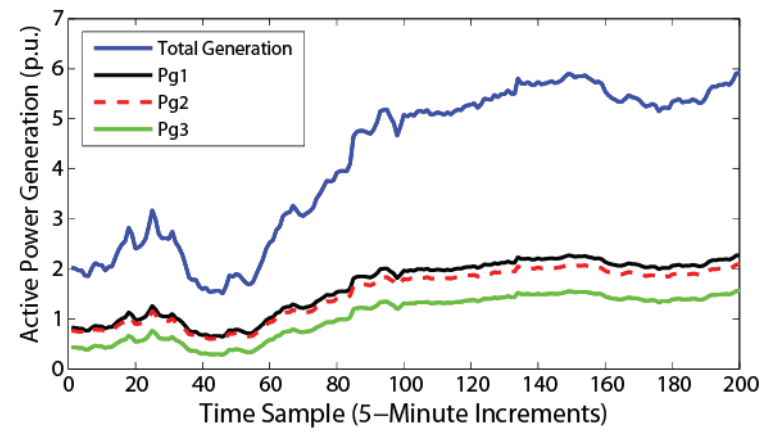

Fig. 6. Optimal generation for $\mathrm{N}=1$ (no storage usage)

multipliers for the coupling constraints, are exchanged after every iteration.

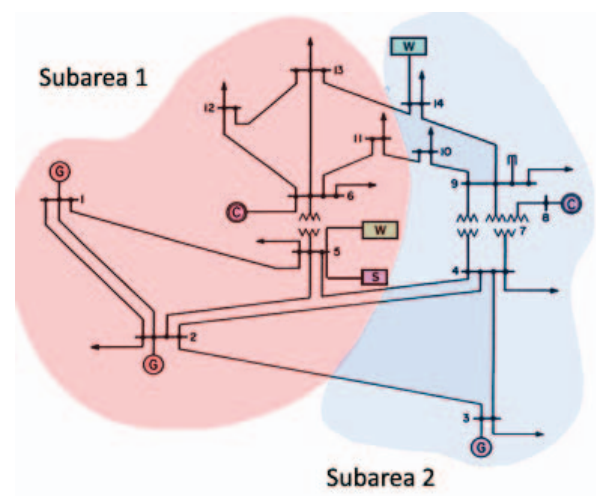

Fig. 7. Decomposed IEEE 14-bus system

$$
\begin{aligned}
& p=1:\{1,2,5,6,11,12,13\} \\
& p=2:\{3,4,7,8,9,10,14\}
\end{aligned}
$$

The optimal generation and energy in storage determined by using the proposed approach for horizons $1,5,8$, and 10 is shown in the following Figures 8 - 13 .

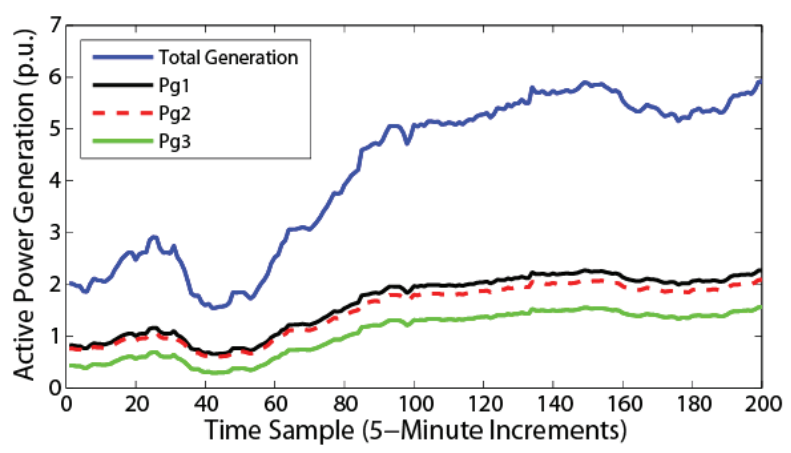

Fig. 8. Optimal generation for $\mathrm{N}=5$

By zooming in to focus on $t=0 \ldots 50$ in Figure 14, the peak-smoothing effect of storage utilization can be seen more clearly. When the algorithm detects that the demand is sharply increasing or wind generation varies rapidly, it realizes that it is more optimal to put energy into storage early to utilize at

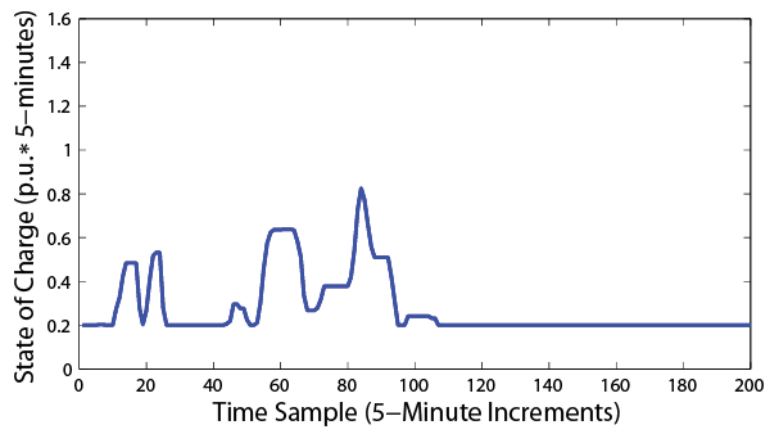

Fig. 9. Optimal state of charge for $\mathrm{N}=5$

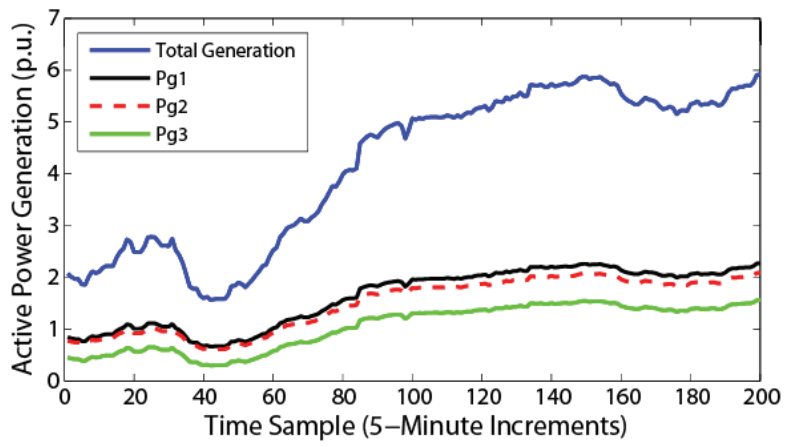

Fig. 10. Optimal generation for $\mathrm{N}=8$

a later time to avoid the fast ramping of these generators. For longer horizons, this smoothing effect becomes more evident.

It is clear that the utilization of storage helps keep generation levels more constant, limiting the ramping up/down of the generators. As the length of the prediction horizon increases, this ramping is limited further. However, for longer horizons, the wind predictions may or may not be completely accurate as assumed here, and the introduction of this error dictates that this trend will not necessarily continue. An increase in storage size will be beneficial in this case.

\section{B. Convergence Rates of Centralized vs. Distributed}

As with many gradient-based optimization algorithms, if the step size in the Newton-Raphson update is too large, the algorithm may diverge from the optimal solution. A damping factor was implemented for the variable update for all subproblems $p \in\{1, \ldots, P\}$ :

$$
x_{p}^{k+1}=x_{p}^{k}-\gamma J_{p}^{-1} K K T_{p}^{k} .
$$

Trials for $\gamma=\{1,0.75,0.5,0.25,0.1\}$ were performed until the algorithm reached convergence. Only in $17.25 \%$ of the cases in the simulations required damping, however; and only $0.0187 \%$ of those cases required heavier damping than 0.75 . The convergence rates are shown in Figures $15-18$ for the centralized and distributed cases using various horizons for an arbitrarily chosen time sample in the simulation $(t=10)$, which is a sample that demonstrates typical performance observed over the entire simulation. The distance from the 


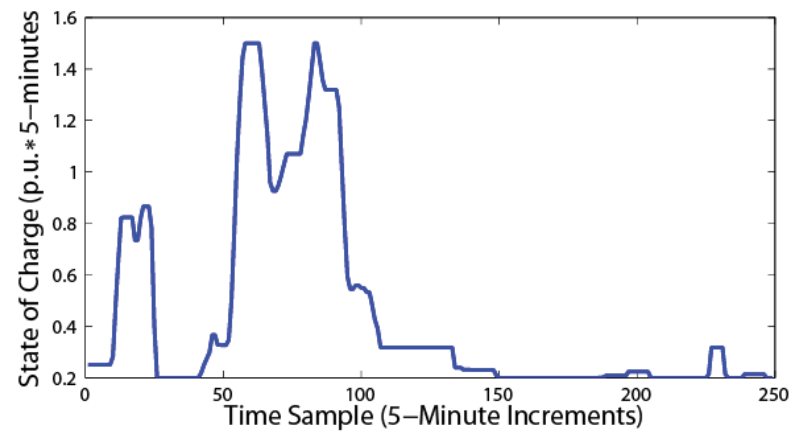

Fig. 11. Optimal state of charge for $\mathrm{N}=8$

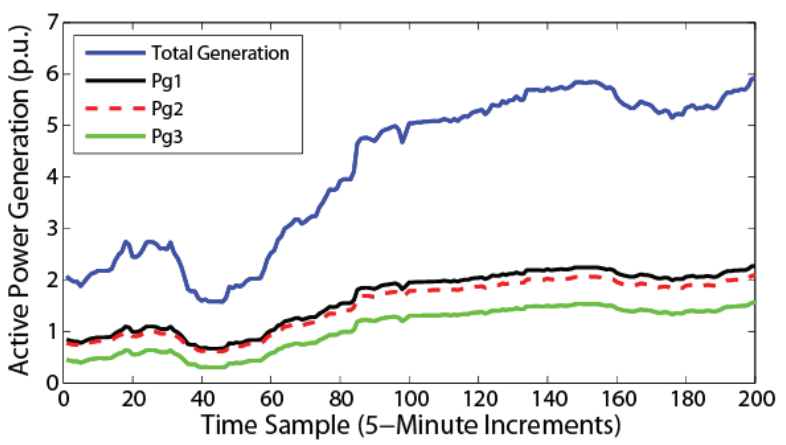

Fig. 12. Optimal generation for $\mathrm{N}=10$

solution is measured in terms of the sum of the Euclidean norm of the $K K T_{p}$ vectors for all subproblems $p \in\{1, \ldots, P\}$ :

$$
\text { Distance From Solution }=\sum_{p=1}^{P}\left\|K K T_{p}\right\|_{2} .
$$

It is clear that as the number of variables in the optimization increases, it becomes more difficult for the optimization to converge, and the discrepancy between the centralized and distributed convergence rates grows. However, it is important to note that this does not indicate anything about computation time. An advantage of the distributed algorithm is that the subproblems can perform their Newton-Raphson steps in parallel and the computational complexity of calculating this update for each subproblem is far less than that needed for the centralized problem.

\section{Accuracy of Results}

The optimal generation values calculated were compared in MATLAB (TOMLAB) using the SNOPT solver [15]. The results indicate that the distributed algorithm presented in this paper converges to the centralized solution calculated using a commercial solver.

\section{CONClusion AND Future Work}

The method we have developed in this paper shows promising results for enhancing the integration of intermittent resources. The solution achieved with decomposition into two control areas is the same as the centralized solution achieved

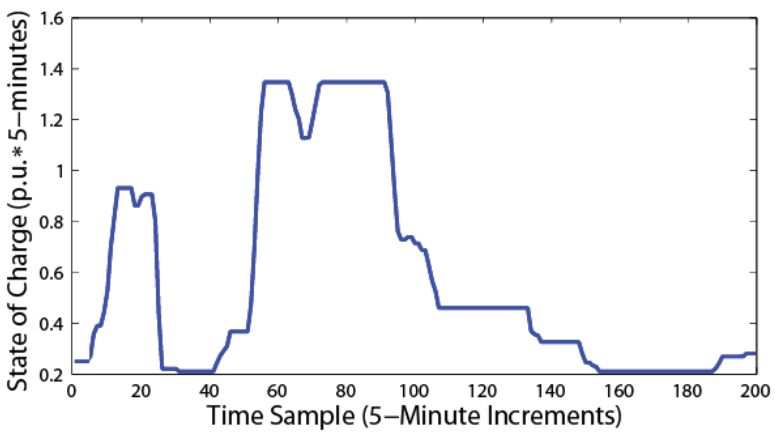

Fig. 13. Optimal state of charge for $\mathrm{N}=10$

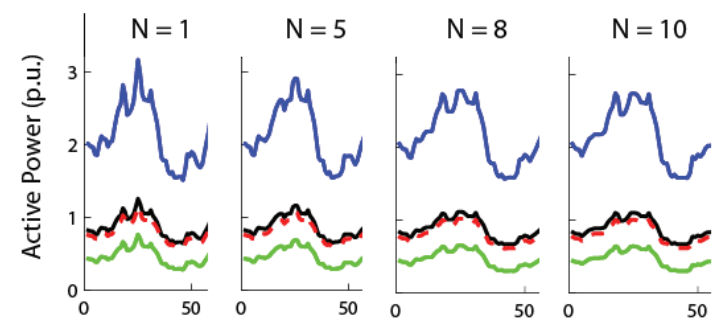

Fig. 14. Smoothing effect with longer horizons

by a commercial solver. By decomposing the system, a better representation of physical control areas is achieved and subproblem computations can easily be parallelized. Because performing one Newton-Raphson iteration before exchanging subproblem data is sufficient, computations are also expedited. Utilization of storage and predictive control provides the benefit of reducing overall generation costs and harmful emissions, and effectively increases the penetration of renewable energy sources.

Future work will focus firstly on making the algorithm more robust. For some load and wind data, especially for longer horizons, the algorithm may fail to converge at certain time samples. The specific conditions under which this happens will be further investigated and a solution for such cases will be determined. After these cases have been accounted for, we will make efforts to determine what the optimal size of storage and backup generation should be for a given level of intermittent energy penetration. This is important for the planning of future electric power systems in the sense that we want to avoid overdesigning the system while still maintaining a secure operation of the system. Finally, with these objectives in mind, we will move to simulations using larger power systems.

\section{ACKNOWLEDGEMENT}

This work was supported by the National Science Foundation under award ECCS 1027576 . The authors are very grateful for this financial assistance that made this project a possibility. 


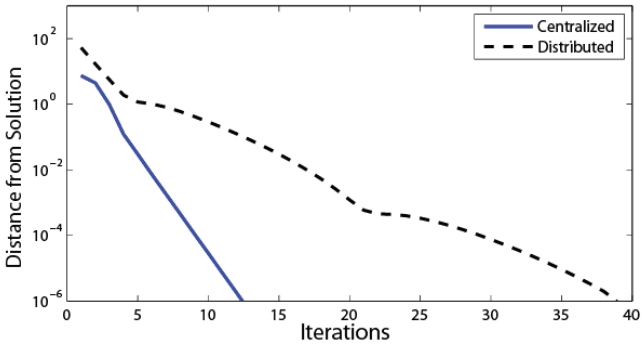

Fig. 15. Convergence rate for $\mathrm{N}=1$

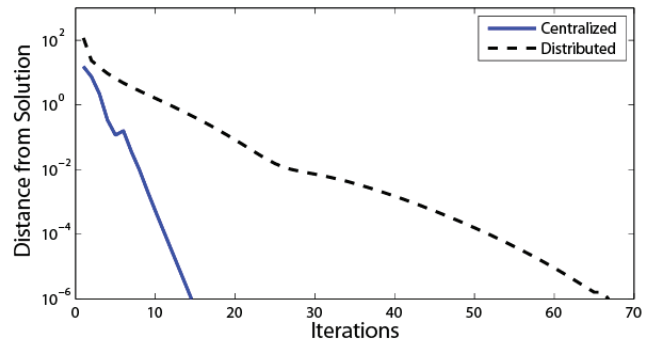

Fig. 16. Convergence rate for $\mathrm{N}=5$

\section{REFERENCES}

[1] U.S. Department of Energy, 20\% Wind Energy by 2030 - Increasing Wind Energy's Contribution to U.S. Electricity Supply. 2008.

[2] Maciejowski, J.M., Predictive Control with Constraints. 2002: Prentice Hall.

[3] R. Findeisen and F. Allgower, An introduction to nonlinear model predictive control, in Proc. 21st Benelux Meeting System and Control, Veldhoven, 2002, pp. 1-23.

[4] Nogales, F.J., A.J. Conejo, and F.J. Prieto, A Decomposition Methodology Applied to the Multi- Area Optimal Power Flow Problem. Annals of Operations Research, 2003. Vol. 120: pp. 99-116.

[5] G. Tognola and R. Bacher, Unlimited point algorithm for OPF problems, IEEE Trans. Power Syst., vol. 14, pp.1046 - 1054 , 1999.

[6] Power System Test Case Archive, http://www.ee.washington.edu/research/pstca/pf14/pg_tca14bus.htm. Last accessed on August 14, 2011.

[7] H. W. Kuhn and A. W. Tucker, Nonlinear programming, in Proc. 2nd Berkeley Symp. Mathematical Statistics and Probability, Berkeley, CA, 1951, pp. 481-492.

[8] Xie, L. and M.D. Ilic. Model predictive dispatch in electric energy systems with intermittent resources. in IEEE International Conference on Systems, Man and Cybernetics, 2008.

[9] Camponogara, E., Jia, D., Krogh, B.H., Talukdar, S., Distributed model predictive control. IEEE Control Systems Magazine, 2002. Vol. 22(1): pp. 44-52.

[10] Talukdar, S., Dong, J., Hines, P., Krogh, B.H., Distributed Model Predictive Control for the Mitigation of Cascading Failures. in 44th IEEE Conference on Decision and Control and European Control Conference. CDC-ECC '05. 2005.

[11] Venkat, A.N., Hiskens, I., Rawlings, J.B., Wright, S.J., Distributed MPC Strategies With Application to Power System Automatic Generation Control. IEEE Transactions on Control Systems Technology, 2008. vol. 16(6): pp. 1192-1206.

[12] G. Hug-Glanzmann and G. Andersson, "Decentralized Optimal Power Flow Control for Overlapping Areas in Power Systems", IEEE Transactions on Power Systems, vol. 24, pp. 327-336, Feb 2009.

[13] H. El Fawal, D. Georges, and G. Bornard, "Optimal control of complex irrigation systems via decomposition-coordination and the use of augmented Lagrangian," in Proc. 1998 IEEE Int. Conf. Systems, Man and Cybernetics, vol. 4, San Diego, CA, 1998, pp. 3874-3879.

[14] Bonneville Power Administration Wind Generation Forecast, http://transmission.bpa.gov/business/operations/wind/forecast/forecast.aspx. Last accessed on August 14, 2011.

[15] Holmstrm, K., Gran, A.O., Edvall, M.M.: Users Guide for TOMLAB/SNOPT, 2008.

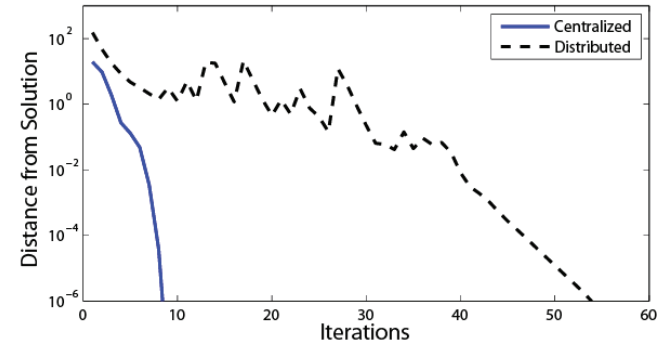

Fig. 17. Convergence rate for $\mathrm{N}=8$

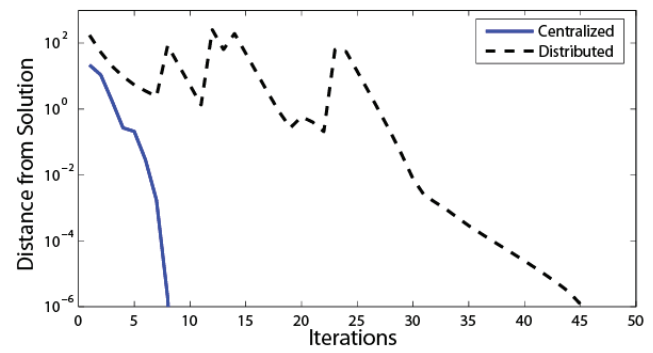

Fig. 18. Convergence rate for $\mathrm{N}=10$

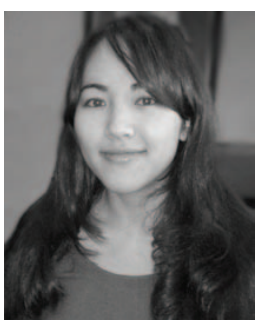

Kyri Baker (S'08) received her B.S. and M.S. in Electrical and Computer Engineering at Carnegie Mellon University in 2009 and 2010. She is currently pursuing her Ph.D. at Carnegie Mellon University. Her research interests include power system optimization and control, smart grid technologies, and renewable energy integration.

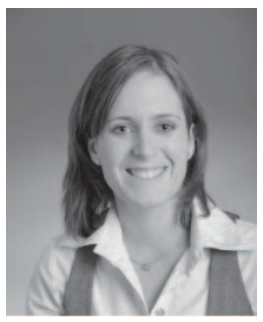

Gabriela Hug ( $\mathrm{S}^{\prime} 05-\mathrm{M}^{\prime} 08$ ) was born in Baden, Switzerland. She received the M.Sc. degree in electrical engineering from the Swiss Federal Institute of Technology (ETH), Zurich, Switzerland, in 2004 and the Ph.D. degree from the same institution in 2008. After her Ph.D., she worked in the Special Studies Group of Hydro One in Toronto, Canada and since 2009 she is an Assistant Professor at Carnegie Mellon University in Pittsburgh, USA. Her research is dedicated to control and optimization of electric power systems.

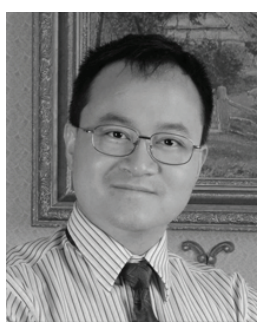

Xin Li (S'01-M'06-SM'10) received the Ph.D. degree in Electrical \& Computer Engineering from Carnegie Mellon University in 2005. He is currently an Assistant Professor in the ECE Department at Carnegie Mellon. Since 2009, he has been appointed as the Assistant Director for FCRP Focus Research Center for Circuit \& System Solutions. His research interests include computer-aided design, neural signal processing, and power system analysis and design. Dr. Li received two ICCAD Best Paper Awards in 2004 and 2011 and one DAC Best Paper Award in 2010 . 